\title{
Clinical evaluation of Oxoid Brilliance MRSA Agar in comparison with bioMérieux MRSA ID medium for detection of livestock-associated meticillin-resistant Staphylococcus aureus
}

\author{
Correspondence \\ Erwin Verkade \\ everkade@amphia.nl
}

Received 4 May 2010

Accepted 10 March 2011

\section{Erwin Verkade, ${ }^{1,2}$ Marianne Ferket ${ }^{1}$ and Jan Kluytmans ${ }^{1,2,3}$}

\author{
${ }^{1}$ Laboratory for Microbiology and Infection Control, Amphia Hospital, Breda, The Netherlands \\ ${ }^{2}$ Laboratory for Medical Microbiology and Immunology, St Elisabeth Hospital, Tilburg, \\ The Netherlands \\ ${ }^{3}$ Department of Medical Microbiology and Infection Prevention, VU University Medical Center, \\ Amsterdam, The Netherlands
}

\begin{abstract}
Oxoid Brilliance MRSA Agar and bioMérieux MRSA ID medium were evaluated for their ability to identify meticillin-resistant Staphylococcus aureus (MRSA) in clinical samples. Nasal and throat samples $(n=629)$ were taken from veterinarians and their household members. The sensitivities of Brilliance MRSA Agar and MRSA ID medium after $20 \mathrm{~h}$ of incubation were 63.6 and $64.5 \%$, and the specificities were 94.1 and $99.4 \%$, respectively. After an enrichment step, the sensitivities increased to 96.3 and $97.2 \%$, but the specificities decreased to 88.7 and $98.5 \%$, respectively. Brilliance MRSA Agar and MRSA ID medium are both sensitive methods for the screening of MRSA in combination with broth enrichment, but positive results require confirmation.
\end{abstract}

\section{INTRODUCTION}

Meticillin-resistant Staphylococcus aureus (MRSA) has emerged worldwide as a nosocomial pathogen of major importance, and the incidence of infections caused by MRSA continues to increase (Archer, 1998; Noskin et al., 2007). Recently, MRSA has emerged in the community (Kluytmans-Vandenbergh \& Kluytmans, 2006). A new clone has also been identified that is related to an extensive reservoir in animals. Persons who are in direct contact with pigs frequently carry livestock-associated MRSA. These isolates belong mainly to multilocus sequence type 398 (ST398) (van Loo et al., 2007; Voss et al., 2005). The extension of MRSA beyond its known boundaries poses an additional challenge for microbiological laboratories to improve their screening strategies. Screening for MRSA among various human populations with increased risk for carriage is an essential component of MRSA control strategies. This includes both patients and healthcare workers (Muto et al., 2003). In human healthcare settings, different procedures employed for the detection of MRSA from clinical samples have variable results (Brown et al., 2005). Methods to detect MRSA in clinical samples ideally should have a high sensitivity and specificity combined with a short time to reporting of the results. To identify S. aureus

Abbreviations: MRSA, meticillin-resistant Staphylococcus aureus; MSSA, meticillin-sensitive $S$. aureus. from contaminated samples more easily and reliably, selective media have been developed (Merlino et al., 2000).

The purpose of this study was to evaluate the sensitivity and specificity of a selective medium, Oxoid Brilliance MRSA Agar, for the identification of MRSA, using nose and throat samples taken from veterinarians and their household members in an ongoing study.

\section{METHODS}

Oxoid Brilliance MRSA Agar is a chromogenic medium for identification of MRSA in human specimens and was supplied as prepared culture plates from Thermo Fisher Scientific. The composition of the chromogenic and selective mix is proprietary. MRSA isolates form distinctive denim-blue colonies on Brilliance MRSA Agar. The selective mixture inhibits meticillin-susceptible $S$. aureus (MSSA) strains, most bacteria not belonging to the genus Staphylococcus and yeasts. Results can be read after $18 \mathrm{~h}$, according to the manufacturer.

Nose and throat samples were taken from veterinarians who worked mainly with pigs. The household members were also sampled and the samples were subsequently sent by mail in a transport container with Amies medium (Transwab; Medical Wire \& Equipment) to the Laboratory for Microbiology and Infection Control in the Amphia Hospital, The Netherlands. A total of 629 freshly collected swabs from a total of 318 patients were analysed in this study. Samples were inoculated onto both Brilliance MRSA Agar and MRSA ID medium (bioMérieux), and diluted with a sterile loop using a three-streak dilution method. In addition, broth enrichment comprising MuellerHinton broth supplemented with $6.5 \% \mathrm{NaCl}$ was carried out using 
the same swabs. From the overnight broth enrichment, subcultures were inoculated onto Brilliance MRSA Agar and MRSA ID medium. The directly inoculated and overnight enriched inoculated plates were read separately after $18-24 \mathrm{~h}$ incubation at $35-37^{\circ} \mathrm{C}$ by different laboratory technicians on different benches. The procedure was performed as recommended by the manufacturer. Growth of colonies showing blue coloration on Brilliance MRSA Agar plates or green coloration on MRSA ID agar plates was considered to be indicative of MRSA. No growth, or colonies with colours other than blue or green, were considered negative. Presumptive $S$. aureus colonies were initially identified by a latex agglutination test (Staphaurex Plus; Thermo Fisher Scientific) and by the detection of DNase (Oxoid DNase agar; Thermo Fisher Scientific). If the results of these tests were discordant, isolates were identified by detection of free coagulase using a tube coagulase test with rabbit plasma. When isolates were identified as $S$. aureus, their meticillin susceptibility was determined using a cefoxitin disc diffusion test according to Clinical and Laboratory Standards Institute standards (NCCLS, 1997). All cefoxitin-resistant isolates were confirmed to be MRSA using a duplex PCR for the mecA and coagulase genes, as described previously (Kluytmans et al., 2002; van Griethuysen et al., 1999), and this was considered the 'gold standard'. When MRSA was recovered from one or more of the media, the sample was considered positive.

\section{RESULTS AND DISCUSSION}

A total of 629 freshly collected nasal and throat samples were analysed in this study. In total, there were 318 nose samples and 311 throat samples. When combined results for all media were analysed, 107 of the 629 samples (17.0\%) harboured MRSA. The presence of MRSA in nose samples was comparable with that in throat samples [60/ $318(18.9 \%)$ vs $47 / 311$ (15.1\%), respectively; $P=0.243$ ]. The prevalence of MRSA in persons with direct contact with pigs was significantly higher than in persons who had not had contact with pigs $[52 / 86(60.5 \%)$ vs $18 / 232$ $(8.4 \%) ; P<0.001]$. Of the 168 samples obtained from veterinarians, $83(49.4 \%)$ harboured MRSA. In contrast, only 24 of the $461(5.2 \%)$ samples from the household members were positive for MRSA. There were no significant differences in the performance of the two chromogenic agar plates with regard to specimen type. The results obtained with direct inoculation of the media and after broth enrichment are shown in Table 1. The sensitivity of directly inoculated Brilliance MRSA Agar was comparable with that of directly inoculated MRSA ID medium [68/107 (63.6\%) vs 69/107 (64.5\%), respectively; $P=1.0]$. However, the specificity of directly inoculated Brilliance MRSA Agar was significantly lower than that of MRSA ID medium [491/522 (94.1\%) vs 519/522 (99.4\%), respectively; $P<0.001]$. The negative predictive values of directly inoculated Brilliance MRSA and MRSA ID medium were comparable [491/530 (92.6\%) vs 519/557 (93.2\%), respectively; $P=1.0]$. The positive predictive value of directly inoculated Brilliance MRSA Agar was significantly lower than that of MRSA ID medium [68/99 (68.7\%) vs $69 / 72$ (95.8\%), respectively; $P<0.001$ ]. The addition of broth enrichment resulted in a substantial increase in the yield of MRSA for both agar plates. Brilliance MRSA Agar recovered 103 MRSA strains, resulting in a sensitivity of $96.3 \%$. This was comparable to the sensitivity of MRSA ID medium after broth enrichment [104/107 (97.2\%); $P=1.0]$. The highest specificity following enrichment was achieved by MRSA ID medium (98.5\%), which yielded only eight false-positive results. The number of false-positives was much higher for Brilliance MRSA Agar (59 false-positives), resulting in a specificity of $88.7 \%(P<0.001)$. The colours of the colonies ranged from light blue to the specific denimblue coloration considered typical for Brilliance MRSA Agar. In combination with broth enrichment, the negative predictive value of Brilliance MRSA Agar was comparable to that of MRSA ID medium [463/467 (99.1\%) vs 514/517 (99.4\%), respectively; $P=1.0]$. However, the positive predictive value of Brilliance MRSA Agar was significantly lower than that of MRSA ID medium following enrichment $[103 / 162(63.6 \%)$ vs $104 / 112 \quad(92.9 \%)$, respectively; $P<0.001]$.

Several studies have recently evaluated various novel chromogenic selective media for the detection of MRSA from clinical samples (Malhotra-Kumar et al., 2010; Nonhoff et al., 2009; Peterson et al., 2010). Comparison of the results of these studies is not easy because of differences in the study designs: swab type, body site sampled, direct inoculation versus prior homogenization in saline or broth, incubation time and the use of broth enrichment. In the present study, we evaluated the performance of Brilliance MRSA Agar with and without

Table 1. Analytical performance of Brilliance MRSA Agar and MRSA ID medium in a prospective clinical study of nasal and throat samples ( $n=629$, including $n=107$ MRSA-positive samples)

TP, True-positive; FP, false-positive; TN, true-negative; FN, false-negative; PPV, positive predictive value; NPV, negative predictive value.

\begin{tabular}{|llcccccccc|}
\hline \multirow{2}{*}{ Medium } & \multicolumn{1}{c}{ Method } & TP & FP* & TN & FN & Sensitivity (\%) & Specificity (\%) & PPV (\%) & NPV (\%) \\
\hline \multirow{2}{*}{ Brilliance MRSA } & Direct only & 68 & 31 & 491 & 39 & 63.6 & 94.1 & 68.7 & 92.6 \\
& Direct + enrichment & 103 & 59 & 463 & 4 & 96.3 & 88.7 & 63.6 & 99.1 \\
\multirow{2}{*}{ MRSA ID } & Direct only & 69 & 3 & 519 & 38 & 64.5 & 99.4 & 95.8 & 93.2 \\
& Direct + enrichment & 104 & 8 & 514 & 3 & 97.2 & 98.5 & 92.9 & 99.4 \\
& & & & & & & & \\
\end{tabular}

${ }^{\star}$ False-positive indicates all colonies showing blue coloration on Brilliance MRSA Agar or colonies showing green coloration on MRSA ID medium that were not confirmed to be MRSA. 
broth enrichment in a population with a high prevalence of MRSA carriage. Brilliance MRSA Agar had a high sensitivity when used in combination with broth enrichment. However, without broth enrichment the sensitivity was significantly lower. This confirms the importance of broth enrichment for the accurate detection of MRSA in clinical samples (Malhotra-Kumar et al., 2010; Nonhoff et al., 2009).

It is difficult to compare our findings with those in other studies as there are few clinical studies that have used Brilliance MRSA Agar. However, other chromogenic media have been studied. For example, a study by Nonhoff et al. (2009) evaluated the performance of MRSA ID, MRSAScreen and MRSASelect in combination with broth enrichment for the detection of MRSA in 1002 mucocutaneous swabs from 639 hospitalized patients. Swabs were plated on the three chromogenic media. Subsequently, broth enrichment subculture was carried out by overnight incubation on the different agar plates. MRSA strains were isolated from $68(6.8 \%)$ specimens from 44 patients. The sensitivity of all the chromogenic media was $<50 \%$ after $18 \mathrm{~h}$ incubation but increased with prolonged incubation of $42 \mathrm{~h}$ to $75,80.9$ and $72.1 \%$ for MRSA ID, MRSA-Screen and MRSASelect, respectively. The difference in sensitivity between MRSA-Screen and MRSASelect after $42 \mathrm{~h}$ was significant. After enrichment, the sensitivity for all media was $85.3 \%$ at $24 \mathrm{~h}$ incubation. The specificity was excellent for MRSA ID (99.9\%) and MRSASelect (100\%) after $18 \mathrm{~h}$ and lower for MRSA-Screen (97.2\%), although this was not statistically significant.

Another recently published study that reported similar findings was performed by Malhotra-Kumar et al. (2010). They evaluated and compared the potential for MRSA detection of five chromogenic media - Brilliance MRSA Agar (Oxoid), ChromID (bioMérieux), MRSASelect (BioRad), CHROMagar (CHROMagar-Microbiology) and BBL-CHROMagar (BD Diagnostics). The media were tested on $\log$ serial dilutions of pure isolates of MRSA $(n=60)$, non-MRSA $(n=27)$ and defined mixtures simulating clinical samples $(n=84)$. Further evaluations were carried out on pre-enriched nasal and groin screening swabs $(n=213)$ from 165 hospitalized patients. The cumulative mean sensitivity on isolates, mixtures and clinical samples was highest for Brilliance (97\%) and similar for the other four media ( $\geqslant 92 \%)$. The cumulative mean specificity was highest for BBL-CHROMagar (99\%), followed by MRSASelect (98\%), CHROMagar (97\%), ChromID (89\%) and Brilliance (86\%). False-positive results were mainly due to meticillin-resistant Staphylococcus epidermidis. The two studies described above confirm the additional yield of broth enrichment and are comparable to our findings.

In contrast, Peterson et al. (2010) reported sensitivities that were different from our findings. They compared the performance of Brilliance MRSA Agar with traditional media in detecting MRSA. In this study, 767 nasal samples from a multi-centre study and 667 nasal samples from a tertiary hospital were screened for the presence of MRSA. After $24 \mathrm{~h}$ of incubation, the sensitivity and specificity were as follows: (i) multi-centre study: Brilliance MRSA Agar, 95.4 and $99.7 \%$; tryptic soy agar with $5 \%$ sheep blood, 93.6 and $100 \%$; (ii) tertiary hospital: Brilliance MRSA Agar, 95.2 and 99.5\%; mannitol salt agar, 88.7 and 94.0\%, respectively. Extending the incubation to $48 \mathrm{~h}$ did not significantly improve the recovery of MRSA and resulted in decreased specificity. The higher sensitivity found in this study can easily be explained by the lack of broth enrichment in this comparison. However, this cannot explain the much higher specificity. The discrepancy between the sensitivities obtained in the above-mentioned study in comparison with the direct plating in our study is most probably caused by the addition of broth enrichment in our culture procedure. The differences in performance values may be predominantly due to the use of suboptimal media as the 'gold standard'. Another explanation for the discrepancy between the specificity found in our evaluation and in some other studies may be that we screened a specific population, i.e. veterinarians and their household members, who carry a specific clone of MRSA, named livestock-associated MRSA. Therefore, our results do not necessarily apply to other types of MRSA.

In the present work, the evaluation of the performance of chromogenic media for MRSA detection in screening swab samples in a high-prevalence population demonstrated a low sensitivity (64-65\%) of the two media after 20-24 h of incubation. However, the sensitivity of both media was increased significantly by using an enrichment step.

A selection of the false-positive colonies that grew on the Brilliance MRSA Agar were identified. Sixty per cent belonged to the genus Bacillus, $24 \%$ were coagulasenegative staphylococci and $16 \%$ were MSSA. After performing the additional identification tests recommended by the manufacturers, specificity was improved significantly.

The results of our study have one important limitation: we did not use a non-selective blood agar plate as the 'gold standard'. This could have increased the number of MRSA isolates recovered from the samples. However, this would have resulted in even lower sensitivities compared with the current evaluation.

Despite commercial efforts to develop new media with high sensitivity, the present study, as well as others (MalhotraKumar et al., 2010; Nonhoff et al., 2009), showed a substantial increase in sensitivity following enrichment compared with direct cultures. Therefore, broth enrichment remains necessary for reliable MRSA detection. Indeed, in our study, if screening samples had only been plated directly and inspected after 20-24 h, as suggested by the manufacturers, $29.9 \%$ of the MRSA-positive samples detected would have been missed. In contrast, only one MRSA-positive sample $(0.9 \%)$ would have been missed if samples had been grown exclusively after enrichment. 
In summary, Brilliance MRSA Agar performed similarly to MRSA ID medium with respect to sensitivity but with a significantly lower specificity with and without broth enrichment. An advantage of Brilliance MRSA Agar is that this medium is less light sensitive than MRSA ID medium, making it easier to work with in the laboratory. The additional yield of the broth enrichment was comparable to what has been reported previously and has to be considered as the standard for screening of MRSA.

In conclusion, Brilliance MRSA Agar in combination with broth enrichment is a sensitive method for MRSA detection in people exposed to livestock. However, the specificity is relatively low and suspected colonies need to be confirmed by further testing.

\section{ACKNOWLEDGEMENTS}

The chromogenic medium Oxoid Brilliance MRSA Agar was kindly provided by Thermo Fisher Scientific (Basingstoke, UK). J. K. has received research grant support from Thermo Fisher Scientific and bioMérieux.

\section{REFERENCES}

Archer, G. L. (1998). Staphylococcus aureus: a well-armed pathogen. Clin Infect Dis 26, 1179-1181.

Brown, D. F. J., Edwards, D. I., Hawkey, P. M., Morrison, D., Ridgway, G. L., Towner, K. J. \& Wren, M. W. D. on behalf of the Joint Working Party of the British Society for Antimicrobial Chemotherapy, Hospital Infection Society and Infection Control Nurses Association (2005). Guidelines for the laboratory diagnosis and susceptibility testing of methicillin-resistant Staphylococcus aureus (MRSA). J Antimicrob Chemother 56, 1000-1018.

Kluytmans, J. A., Van Griethuysen, A., Willemse, P. \& Van Keulen, P. (2002). Performance of CHROMagar selective medium and oxacillin resistance screening agar base for identifying Staphylococcus aureus and detecting methicillin resistance. J Clin Microbiol 40, 2480-2482.

Kluytmans-Vandenbergh, M. F. \& Kluytmans, J. A. (2006). Community-acquired methicillin-resistant Staphylococcus aureus: current perspectives. Clin Microbiol Infect 12 (Suppl. 1), 9-15.
Malhotra-Kumar, S., Abrahantes, J. C., Sabiiti, W., Lammens, C., Vercauteren, G., leven, M., Molenberghs, G., Aerts, M., Goossens, H. \& MOSAR WP2 Study Team (2010). Evaluation of chromogenic media for detection of methicillin-resistant Staphylococcus aureus. J Clin Microbiol 48, 1040-1046.

Merlino, J., Leroi, M., Bradbury, R., Veal, D. \& Harbour, C. (2000). New chromogenic identification and detection of Staphylococcus aureus and methicillin-resistant S. aureus. J Clin Microbiol 38, 23782380.

Muto, C. A., Jernigan, J. A., Ostrowsky, B. E., Richet, H. M., Jarvis, W. R., Boyce, J. M. \& Farr, B. M. (2003). SHEA guideline for preventing nosocomial transmission of multidrug-resistant strains of Staphylococcus aureus and Enterococcus. Infect Control Hosp Epidemiol 24, 362-386.

NCCLS (1997). Methods for Dilution Antimicrobial Susceptibility Tests for Bacteria that Grow Aerobically, 4th edn. Approved Standard. M7A4. Wayne, PA: National Committee for Clinical Laboratory Standards.

Nonhoff, C., Denis, O., Brenner, A., Buidin, P., Legros, N., Thiroux, C., Dramaix, M. \& Struelens, M. J. (2009). Comparison of three chromogenic media and enrichment broth media for the detection of methicillin-resistant Staphylococcus aureus from mucocutaneous screening specimens. Eur J Clin Microbiol Infect Dis 28, 363-369.

Noskin, G. A., Rubin, R. J., Schentag, J. J., Kluytmans, J., Hedblom, E. C., Jacobson, C., Smulders, M., Gemmen, E. \& Bharmal, M. (2007). National trends in Staphylococcus aureus infection rates: impact on economic burden and mortality over a 6-year period (1998-2003). Clin Infect Dis 45, 1132-1140.

Peterson, J. F., Riebe, K. M., Hall, G. S., Wilson, D., Whittier, S., Palavecino, E. \& Ledeboer, N. A. (2010). Spectra MRSA, a new chromogenic agar medium to screen for methicillin-resistant Staphylococcus aureus. J Clin Microbiol 48, 215-219.

van Griethuysen, A. J., Pouw, M., van Leeuwen, N., Heck, M., Willemse, P., Buiting, A. \& Kluytmans, J. (1999). Rapid slide latex agglutination test for detection of methicillin resistance in Staphylococcus aureus. J Clin Microbiol 37, 2789-2792.

van Loo, I., Huijsdens, X., Tiemersma, E., de Neeling, A., van de Sande-Bruinsma, N., Beaujean, D., Voss, A. \& Kluytmans, J. (2007). Emergence of methicillin-resistant Staphylococcus aureus of animal origin in humans. Emerg Infect Dis 13, 1834-1839.

Voss, A., Loeffen, F., Bakker, J., Klaassen, C. \& Wulf, M. (2005). Methicillin-resistant Staphylococcus aureus in pig farming. Emerg Infect Dis 11, 1965-1966. 